\title{
Induction of a Salt Appetite Alters Dendritic Morphology in Nucleus Accumbens and Sensitizes Rats to Amphetamine
}

\author{
Mitchell F. Roitman, Elisa Na, Gregory Anderson, Theresa A. Jones, and llene L. Bernstein \\ Program in Neurobiology and Behavior and Department of Psychology, University of Washington, Seattle, \\ Washington 98195
}

\begin{abstract}
Sensitization to drugs, such as amphetamine, is associated with alterations in the morphology of neurons in the nucleus accumbens, a brain region critical to motivation and reward. The studies reported here indicate that a strong natural motivator, sodium depletion and associated salt appetite, also leads to alterations in neurons in nucleus accumbens. Medium spiny neurons in the shell of the nucleus accumbens of rats that had experienced sodium depletions had significantly more dendritic branches and spines than controls. In addition, a history of
\end{abstract}

sodium depletions was found to have cross-sensitization effects, leading to enhanced psychostimulant responses to amphetamine. Thus, neuronal alterations common to salt and drug sensitization may provide a general mechanism for enhanced behavioral responses to subsequent exposures to these challenges.

Key words: nucleus accumbens; amphetamine; sensitization; salt appetite; Golgi staining; plasticity; psychostimulant drugs
Sensitization is a well documented effect of repeated exposure to drugs such as amphetamine and cocaine (Pierce and Kalivas, 1997). Sensitization represents a form of long-term plasticity that, in important respects, differs from associative learning but may share some common mechanisms (Berke and Hyman, 2000). In the laboratory, sensitization is displayed by increases in the psychostimulant effects of the drugs after multiple previous exposures (Segal and Mandell, 1974; Post and Rose, 1976). Unlike transient drug effects, such as tolerance and withdrawal, sensitization can last as long as 1 year after the last drug administration in rats. The persistence of these effects implicates mechanisms distinct from those responsible for more transient drug effects.

Induction of a salt appetite, a strong natural motivator, is also associated with sensitization (Sakai et al., 1987). Salt appetite, the innate response to sodium need, is expressed as eager ingestion of $\mathrm{NaCl}$ (Richter, 1956; Denton, 1982). Salt appetite is strongly enhanced in rats with a history of previous episodes of sodium depletion. Sodium depletion has been reported to have additional long-term effects on behavior, including an increase in need-free salt intake of the individual (Sakai et al., 1989) and an increase in salt intake of the offspring of individuals depleted of sodium during their pregnancy (Nicolaïdis et al., 1990; Crystal and Bernstein, 1998). Thus, the experience of sodium depletion can lead to durable, possibly life-long, changes in behavioral responses to salt.

Repeated amphetamine treatments, using a delivery schedule known to support sensitization, have been shown to alter the morphology of neurons in the nucleus accumbens, a brain region that plays an important role in motivated behaviors and responses to drugs of abuse (Robinson and Kolb, 1997, 1999). Golgi-stained neurons from rats given multiple amphetamine injections over a

Received Jan. 23, 2002; revised March 6, 2002; accepted March 11, 2002.

This work was supported in part by a University of Washington Royalty Research Fund grant. We thank Todd E. Thiele, Ming Teng Koh, and Ann Voorhies for assistance with these studies.

Correspondence should be addressed to Dr. Ilene L. Bernstein, Department of Psychology, Box 351525, University of Washington, Seattle, WA 98195-1525. E-mail: ileneb@u.washington.edu.

Copyright (C) 2002 Society for Neuroscience $\quad 0270-6474 / 02 / 220001-\bullet \$ 15.00 / 0$ prolonged period, were found to have a significantly greater number of dendritic branches and more dendritic spines compared with controls, morphological changes indicative of synaptic alterations that could underlie behavioral sensitization. The nucleus accumbens is a major projection site of the mesolimbic dopamine system, a system strongly implicated in regulating motivated behaviors not only to artificial rewards, such as drugs of abuse, but also to natural rewards, such as sodium after sodium depletion (Heimer et al., 1993; Roitman et al., 1997, 1999; Kelley, 1999; Lucas et al., 2000).

The present studies assess whether sensitization of salt appetite, like sensitization to amphetamine, is associated with morphological changes by examining dendritic morphology in the nucleus accumbens of rats exposed to repeated sodium depletions. In addition, we evaluated whether a history of sodium depletion yields cross-sensitization effects, namely sensitization to the psychostimulant effects of amphetamine.

\section{MATERIALS AND METHODS}

Male Long-Evans rats weighing between 285 and $415 \mathrm{gm}$ at the start of the experiment were housed individually on a $12 \mathrm{hr}$ light/dark cycle and maintained with access to water and Teklad (Madison, WI) Rodent Chow ad libitum, except as otherwise noted. To induce a salt appetite, animals received sodium-depleting treatments with the diuretic furosemide using a method modified from Wolf (1982) (Midkiff et al., 1987). Furosemide (total dose of $10 \mathrm{mg} / \mathrm{kg}$ ) or isotonic saline was administered

This article is published in The Journal of Neuroscience, Rapid Communications Section, which publishes brief, peerreviewed papers online, not in print. Rapid Communications are posted online approximately one month earlier than they would appear if printed. They are listed in the Table of Contents of the next open issue of JNeurosci. Cite this article as: JNeurosci, 2002, 22:RC225 (1-5). The publication date is the date of posting online at www.jneurosci.org.

http://www.jneurosci.org/cgi/content/full/6416 
in two subcutaneous injections spaced $1 \mathrm{hr}$ apart. Diuresis was confirmed by comparing $3 \mathrm{hr}$ postinjection body weights with preinjection weights, with a criterion for weigh loss of at least $18 \mathrm{gm}$ in furosemide-injected rats and no more than $6 \mathrm{gm}$ for controls. Detailed analysis of effects of this treatment protocol on sodium excretion were obtained from a separate group of animals. Furosemide-treated animals $(n=4)$ excreted $3.59 \pm 66 \mathrm{mmol} \mathrm{Na}{ }^{+}$per kilogram of body weight during the $24 \mathrm{hr}$ after injection compared with saline-treated controls $(n=3)$, which excreted $0.66 \pm 17 \mathrm{mmol} \mathrm{Na}{ }^{+}$per kilogram of body weight during the same period. Three hours after injections, rats were given sodium-deficient chow (ICN Nutritional Biochemicals, Cleveland, $\mathrm{OH}$ ) and distilled water. Twenty-four hours later, food and water were removed, and rats were offered $3 \% \mathrm{NaCl}$ solution for $1 \mathrm{hr}$. After testing, water and standard chow were returned. One week later, depletion and testing were repeated.

In the first study, assessing Golgi-stained material, the rats were depleted for a third time after an interval of 1 week. Twenty-four hours later, all rats were given a lethal dose of sodium pentobarbitol. Groups included depleted $(n=7)$ and control $(n=5)$ animals. Rats were perfused, and brains were removed, blocked for the nucleus accumbens, and placed in Golgi-Cox solution for 6 weeks. After standard reaction and dehydration procedures (Ramon-Moliner, 1970), brain blocks were embedded in epoxy resin, and $200 \mu \mathrm{m}$ coronal sections were cut throughout the rostrocaudal extent of the nucleus accumbens.

To select and quantify nucleus accumbens neurons for analysis, the boundaries of the nucleus were drawn using Neurolucida software (MicroBrightField, Colchester, VT) and atlas templates (Paxinos and Watson, 1986). Neurons were selected and analyzed by an observer unaware of experimental condition. Analyses focused on the shell region of the nucleus accumbens because this is the region considered to have the strongest connections with the limbic system (Heimer et al., 1993) and because previous work (Pierce and Kalivas, 1995; Robinson and Kolb, 1997, 1999) provided evidence of plasticity in this region after sensitization. Criteria for selection of neurons were as follows: (1) darkly and completely stained cell bodies; (2) dendritic processes that could be clearly followed to their terminal tips; (3) no beading or interruptions in dendritic processes along the way to the terminal tips; and (4) no obstruction of dendritic processes by astrocytes, blood vessels, or the processes of neighboring neurons. Neurons were traced under magnification, and length measurements were made using NeuroExplorer software (MicroBrightField). For each neuron, the number and centrifugal order of dendritic branches was recorded. Quantification of the order of branches is important because it appears to be more likely for increases in branches to occur at distal (relative to the soma) segments, and distal segments are longer than proximal segments (Greenough and Volkmar, 1973; Greenough and Chang, 1988). For spine counts, third-order terminal segments were chosen and examined under $40 \times$ magnification. The terminal tip $(10 \mu \mathrm{m})$ of the segment was drawn under a $100 \times$ oil objective, and spine counts along this segment were made to yield spine density (number of spines per $10 \mu \mathrm{m}$ length). For dendritic morphology and spine counts, 5-10 neurons per rat were chosen. The values for all neurons per rat were averaged such that a single averaged value for each rat was included in the final analyses.

Because the effects of sodium depletion history and multiple amphetamine treatments on neuronal morphology in nucleus accumbens were similar, we next examined whether the behavioral effects of sodium depletions were also similar, that is, whether they led to sensitization to the psychostimulant effects of amphetamine. Therefore, the second study examined whether a history of sodium depletion leads to crosssensitization, namely an enhancement of the psychomotor stimulant response to amphetamine.

Long-Evans rats received sodium depletions ("history;" $n=9$ ) or sham treatments ("no history;" $n=9$ ). The depletion group was exposed to two sodium depletion treatments and offered $\mathrm{NaCl}$ solution as above. One week after recovery from the second depletion, rats were habituated to a novel open field by being allowed to explore for $30 \mathrm{~min}$ per day for $2 \mathrm{~d}$. The square open field was constructed of opaque black Plexiglas divided into $3 \times 3$ compartments by white tape applied to the floor. Dimensions of the field were 39.25 inches in width and length with a height of 12.00 inches. The psychostimulant effects of amphetamine were then assessed in the same field by placing each rat in the field for a baseline period of $30 \mathrm{~min}$. They were then injected with amphetamine ( $d$-amphetamine sulfate; $2 \mathrm{mg} / \mathrm{kg}$, i.p.; Sigma, St. Louis, MO) and replaced in the field for an additional $30 \mathrm{~min}$. Activity in the field was videotaped for subsequent analysis by an observer blind to depletion history. A line crossing was defined as movement of the
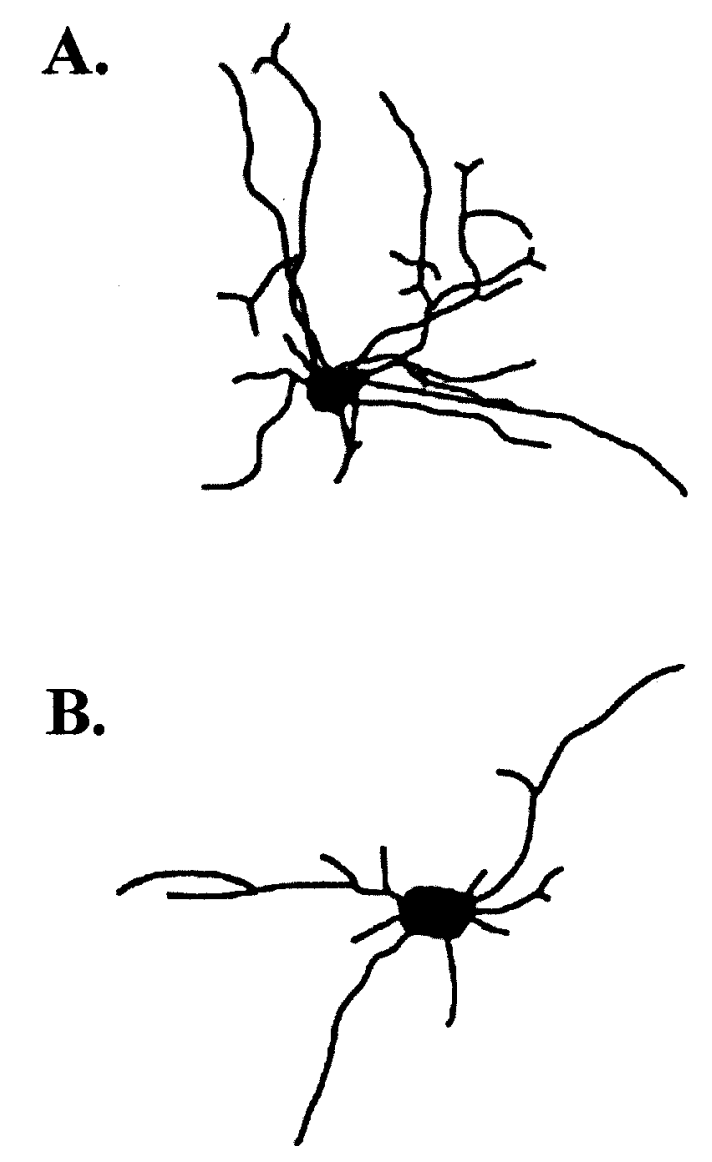

Figure 1. Neurolucida drawings of representative neurons from the shell of the nucleus accumbens of a rat with a history of sodium depletions $(A)$ and a rat with no history of depletions $(B)$. Cells were chosen to reflect the average difference in dendritic length between the two groups.

animal that brought more than one-half of its body over a line, into a different compartment. A rear was defined as a period in which both forepaws were raised and the animal's weight was supported on its hindpaws. Counts were made of each incident of line crossing and rearing in blocks of $10 \mathrm{~min}$. Analyses were performed on the last 10 min of the baseline period and the 30 min after amphetamine administration.

Statistical analyses. Data were analyzed using $t$ tests and ANOVAs. In situations in which multiple $t$ tests were performed on the same data set, the Bonferroni correction procedure was used; the 0.05 level required for statistical significance was divided by the number of tests to yield a more stringent criterion.

\section{RESULTS}

\section{Sodium depletions lead to increases in higher-order dendritic branches in medium spiny neurons of the shell of the nucleus accumbens}

As reported previously (Sakai et al., 1987), $\mathrm{NaCl}$ intake of depleted animals after the first and second depletions showed evidence of sensitization of salt appetite $(10.33 \pm 0.98 \mathrm{ml}$ after second depletion vs $7.8 \pm 1.02 \mathrm{ml}$ after first depletion; $p<0.01$ ).

Significant differences were observed in dendritic morphology of spiny neurons in the shell of the nucleus accumbens in rats with and without a history of sodium depletions (Fig. 1). The total length of dendrites, including all segments, per neuron was significantly greater in depleted rats than in controls (depleted, $789 \pm 27 \mu \mathrm{m}$; control, $\left.584 \pm 76 \mu \mathrm{m} ; t_{(10)}=2.90 ; p<0.05\right)$. Average number of terminal segments per order was determined. No significant differences between groups were found for first- 

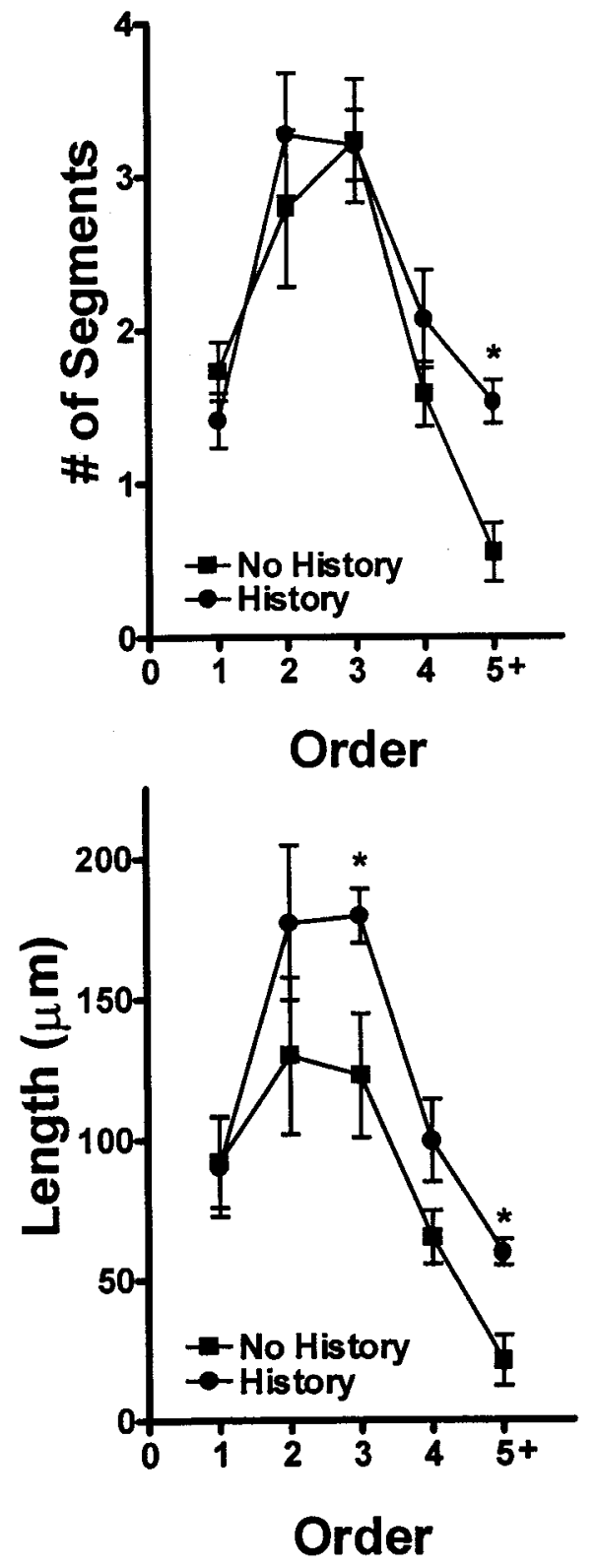

Figure 2. Number and length of dendritic terminal segments, by order, of medium spiny neurons in the shell of the nucleus accumbens in rats with and without a history of sodium depletions. Top, Average \pm SE number of terminal segments per order in rats experiencing multiple sodium depletions (History) and in controls (No History). Order based on an ordering system with each branch arising directly from the soma assigned an order of 1, each bifurcation arising from this branch assigned an order of 2 , and so forth. Bottom, Average \pm SE length of terminal segments per order in rats experiencing multiple sodium depletions and in controls. ${ }^{*} p<0.05$ for history versus no history.

through fourth-order segments. However, there were significantly more terminal segments of fifth- and higher-order in neurons of depleted compared with control rats $\left(t_{(10)}=4.27 ; p<0.01\right.$ ) (Fig. $2)$. Average length of dendritic segments from shell neurons as a function of branch order can be viewed in Figure 2. Differences are evident between depleted and control rats, with these differences appearing in higher-order dendrites (three and above) (third order, $t_{(10)}=3.39, p<0.01$; fifth order, $t_{(10)}=4.5, p<$ 0.01 ). Differences in terminal segment number (five and above) and segment length (third and fifth order) were significant after Bonferroni correction.

Spine density of third-order terminal segments was evaluated, and no differences between groups were found. Spine number at terminal tips is a product of spine density by segment length. Because the length of segments increased and spine density remained stable, this indicates that a complement of spines was added to the added dendritic length.

\section{A history of sodium depletions sensitizes the psychomotor activational effects of amphetamine}

Locomotor activity in the open field during the baseline period and after treatment with amphetamine can be viewed in Figure 3. Horizontal activity (number of line crossings) and rearing episodes were counted, and these scores are depicted in the top and bottom portions of Figure 3, respectively. During the baseline period (before drug injection), numbers of line crossings and rearings appear higher in control than depleted animals. This difference was significant for line crossings $\left(t_{(16)}=2.46 ; p<0.05\right)$ but not for rearing. Increases in line crossings, relative to baseline, occur in both control (no history) (ANOVA; $F_{(3,24)}=8.46$; $p<0.01$ ) and previously depleted (ANOVA; $F_{(3,24)}=15.8 ; p<$ $0.001)$ rats. Differences between the two groups in amount of horizontal activity after amphetamine were not statistically significant. A different pattern was seen when rearing was assessed. Increases in rearing activity over baseline were highly statistically significant in rats with a history of depletion $\left(\mathrm{ANOVA} ; F_{(3,24)}=\right.$ 13.11; $p<0.001$ ) but not in controls (ANOVA; $F_{(3,24)}=1.09 ; p=$ 0.37). Comparing the two groups directly indicated more rearing in the previously depleted rats than in controls, and this difference was statistically significant during the second $10 \mathrm{~min}$ period after amphetamine injection $(p<0.05)$. Together, the rearing data provide evidence of cross-sensitization, with nearly twice as many episodes of rearing in rats with a history of sodium depletion.

\section{DISCUSSION}

The present results provide evidence that a history of sodium depletions is associated with dendritic growth and development of spines in the shell region of the nucleus accumbens. The pattern of dendritic addition, with an increase in topologically higher-order segments, is consistent with previous findings of experience-dependent dendritic growth in adult animals (Greenough and Volkmar, 1973; Greenough and Chang, 1988). The observed alterations are quite similar to those observed after amphetamine sensitization (Robinson and Kolb, 1997). The similarity in pattern of morphological changes seen after amphetamine and salt sensitization suggests that sensitization to a challenge, whether it is a psychoactive drug or acute sodium depletion, is associated with morphological changes in the dendrites of neurons in nucleus accumbens and that these changes are consistent with synaptic reorganization. What is particularly striking about the present findings is that they occurred relatively quickly, that is, 2 weeks after the first sodium-depleting treatment. This contrasts with the 10 week period elapsing in the amphetamine sensitization protocol (Robinson and Kolb, 1997).

Although sodium depletion and amphetamine appear to have similar effects on dendritic morphology in the nucleus accumbens, the pattern of changes is not identical. Notably, Robinson and Kolb (1997) found robust effects on spine density, whereas we found more total spines but no change in spine density. This difference could be attributable to qualitative differences between 

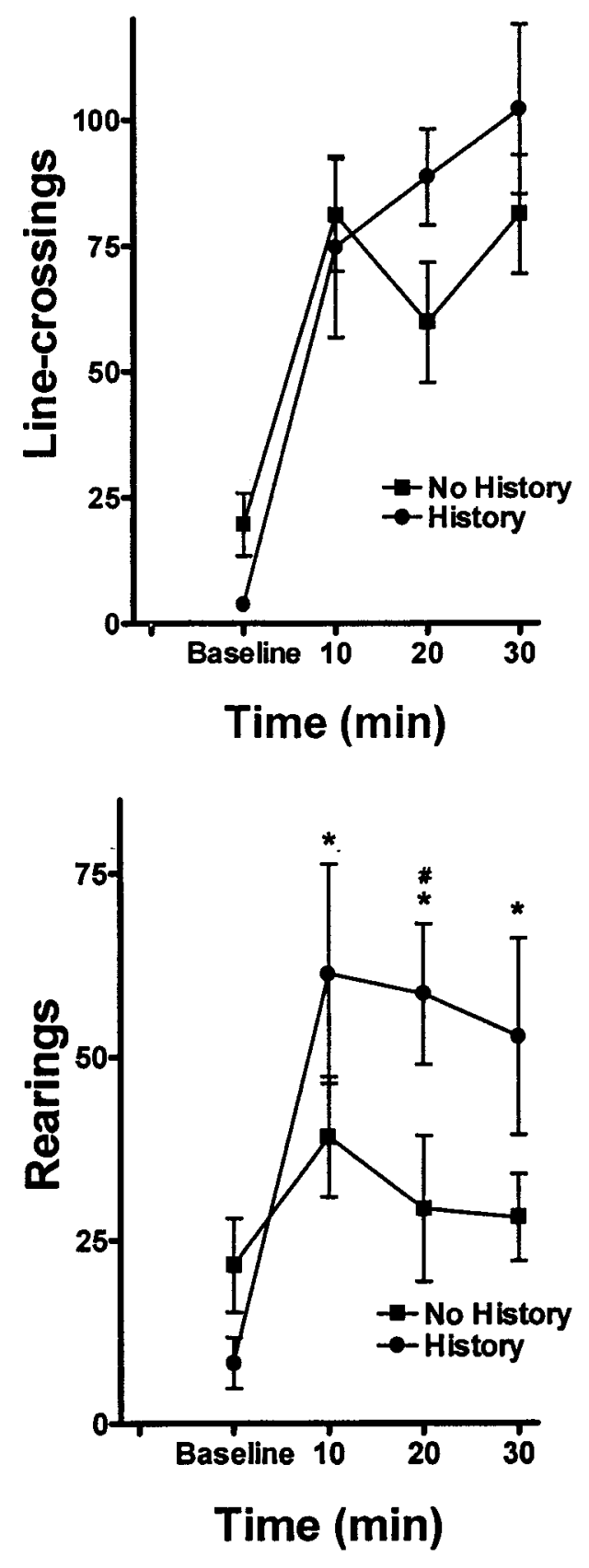

Figure 3. Amphetamine-stimulated activity in an open field in rats with and without a history of sodium depletions. Top, Average \pm SE number of line crossings in the 10 min period before amphetamine administration (Baseline) and in 10 min blocks after administration of amphetamine (2 $\mathrm{mg} / \mathrm{kg}$ ). Bottom, Average \pm SE number of rearings in the $10 \mathrm{~min}$ period before amphetamine administration (Baseline) and in 10 min blocks after amphetamine. ${ }^{*} p<0.05$ relative to baseline; $\# p<0.05$ for history versus no history.

the effects of these treatments, differences in the severity of the treatments, or differences in timing of changes. The observation that the present effects on dendritic length, on a percentage basis $(>30 \%)$, are as great or greater than those seen by Robinson and Kolb (1997) argues against differences in treatment severity as the cause of this difference. Differences between the two studies in time elapsing between treatment and death were considerable, and it is possible that Robinson and Kolb (1997) would have seen a pattern similar to ours if they had looked sooner or we would have seen a pattern like theirs if we had waited longer. It is also possible that these differences reflect genuine differences in the effects of these two types of treatment on synaptic restructuring.

The similarity in the effects of sodium depletion and amphetamine on dendritic morphology in the nucleus accumbens raised the question of whether these two treatments affect the same neuronal populations and whether they have similar behavioral effects. To address this issue, we examined whether sodium depletion would sensitize the locomotor response to amphetamine. Distinct effects on separate neuronal populations would predict that sensitization, after a given treatment, would be restricted to the motivational system that was activated. Alternatively, general amplification within motivational circuits after strong physiological challenges might yield significant sensitization across treatment categories. Support can be found in the recent research literature for both overlap and separation of natural and artificial motive circuits (Cabib et al., 2000; Carelli et al., 2000; Bradley and Meisel, 2001). For example, in a recent report addressing strain differences between mice in their response to amphetamine, DBA/2J, but not C57/BL6J, mice displayed a strong enhancement of their locomotor response to amphetamine after a brief period of food restriction (Cabib et al., 2000). The response to amphetamine was measured after restoration of ad libitum food; apparently, the history of food restriction was sufficient to alter the locomotor response to the drug.

In the present study, rats with a history of sodium depletions displayed more rearing, but not more horizontal movements, in response to amphetamine than did rats without a depletion history. Thus, induction of a strong salt appetite appears to sensitize not only salt appetite but also some of the psychomotor stimulant effects of amphetamine. This is consistent with the proposition that the sodium depletion and amphetamine treatments have similar effects not only on dendritic morphology in nucleus accumbens but also on the behavioral response to amphetamine. It is unclear why effects of depletions were selective, namely an increase in rearing but not horizontal movements, because these two responses are generally viewed as related responses to psychostimulant-induced activation of exploratory behavior. Nonetheless, there have been reports of differential effects of experimental treatments on locomotion and rearing. For example, a clear dissociation between effects of naloxone on amphetamine-induced rearing and locomotion (Balcells-Olivero and Vezina, 1997) suggests that, of the two motor responses, rearing in response to amphetamine is substantially more dependent on opiate mechanisms. With regard to the present findings, the conclusion that amphetamine and sodium depletions have significantly different effects on locomotor sensitization would appear to be premature. Instead, it would be useful to parametrically compare, within our testing protocol, whether effects on rearing and not locomotion are dependent on challenge dose of amphetamine and whether the pattern can be duplicated by any sensitizing regimen of amphetamine.

The loss of sodium caused by furosemide administration is typically restored and exceeded by access to $\mathrm{NaCl}$ solution (Midkiff et al., 1987). Therefore, at the time of testing with amphetamine, the rats had returned to a sodium-replete condition. Thus, the cross-sensitization effects do not require an ongoing need or challenge but rather reflect the consequence of the animals' previous history of deprivation. The results are consistent with the hypothesis that the increases in dendritic length and synapse number, provoked by sodium depletions, may have behavioral consequences beyond the realm of responses to salt.

Interest in drug sensitization has increased in recent years, in 
part because the persistence of sensitization effects provides a parallel to the prolonged susceptibility to relapse of individuals with a history of substance abuse (Pierce and Kalivas, 1997; Berke and Hyman, 2000). The present findings demonstrate that similar changes in dendritic morphology occur after acute sodium depletions and repeated exposure to psychostimulant drugs. Thus, neuronal alterations common to salt and drug sensitization appear to represent a general neuronal response to a strong physiological challenge that may provide a mechanism for an enhanced behavioral response to subsequent exposures to that challenge. The demonstration that acute sodium depletion had cross-sensitization effects, leading to an enhanced psychostimulant response to amphetamine, suggests significant overlap between the affected neuronal populations within nucleus accumbens. Importantly, these results suggest that previous induction of strong natural drives, such as hunger (Cabib et al., 2000) or sodium depletion, can lead to drug sensitization in individuals not previously exposed to those drugs.

\section{REFERENCES}

Balcells-Olivero M, Vezina P (1997) Effects of naltrexone on amphetamine-induced locomotion and rearing: acute and repeated injections. Psychopharmacology 131:230-238.

Berke JD, Hyman SE (2000) Addictions, dopamine, and the molecular mechanisms of memory. Neuron 25:515-532.

Bradley KC, Meisel RL (2001) Sexual behavior induction of c-Fos in the nucleus accumbens and amphetamine-stimulated locomotor activity are sensitized by previous sexual experience in female Syrian hamsters. J Neurosci 21:2123-2130.

Cabib S, Orsini C, Le Moal M, Piazza PV (2000) Abolition and reversal of strain differences in behavioral responses to drugs of abuse after a brief experience. Science 289:463-465.

Carelli RM, Ijames SG, Crumling AJ (2000) Evidence that separate neural circuits in the nucleus accumbens encode cocaine versus "natural" (water and food) reward. J Neurosci 20:4255-4266.

Crystal SR, Bernstein IL (1998) Infant salt preference and mother's morning sickness. Appetite 30:297-307.

Denton DA (1982) The hunger for salt: an anthropological, physiological and medical analysis. Berlin: Springer.

Greenough WT, Chang F-LF (1988) Plasticity of synapse structure and pattern in the cerebral cortex. In: Cerebral cortex, Vol 7 (Jones EG, Peters A, eds), pp 391-440. New York: Plenum.

Greenough WT, Volkmar FR (1973) Pattern of dendritic branching in occipital cortex of rats reared in complex environments. Exp Neurol 40:491-504.

Heimer L, Alheid GF, Zahm DS (1993) Basal forebrain organization: an anatomical framework for motor aspects of drive and motivation. In: Limbic motor circuits and neuropsychiatry (Kalivas PW, Barnes CD, eds), pp 1-32. Boca Raton, FL: CRC.
Kelley A (1999) Functional specificity of ventral striatal compartments in appetitive behaviors. Ann NY Acad Sci 877:71-90.

Lucas LR, Pompei P, McEwen BS (2000) Salt appetite in salt-replete rats: involvement of mesolimbic structures in deoxycorticosteroneinduced salt craving behavior. Neuroendocrinology 71:386-395.

Midkiff EE, Fitts DA, Simpson JB, Bernstein IL (1987) Attenuated sodium appetite in response to sodium deficiency in Fischer-344 rats. Am J Physiol 252:R562-R566.

Nicolaïdis S, Galaverna O, Metzler CH (1990) Extracellular dehydration during pregnancy increases salt appetite of offspring. Am J Physiol 258:R281-R283.

Paxinos G, Watson C (1986) The rat brain in stereotaxic coordinates, Ed 2. Orlando, FL: Academic.

Pierce RC, Kalivas PW (1995) Amphetamine produces sensitized increases in locomotion and extracellular dopamine preferentially in the nucleus accumbens shell of rats administered repeated cocaine. J Pharmacol Exp Ther 275:1019-1029.

Pierce RC, Kalivas PW (1997) A circuitry model of the expression of behavioral sensitization to amphetamine-like psychostimulants. Brain Res Rev 25:192-216.

Post RM, Rose H (1976) Increasing effects of repetitive cocaine administration in the rat. Nature 260:731-732.

Ramon-Moliner E (1970) The Golgi-Cox technique. In: Contemporary methods in neuroanatomy (Nauta WJH, Ebesson SOE, eds), pp 32-47. New York: Springer.

Richter CP (1956) Salt appetite of mammals: its dependence on instinct and metabolism. In: L'Instinct dans le comportement des animaux et de l'homme, p 577. Paris: Masson.

Robinson TE, Kolb B (1997) Persistent structural modifications in nucleus accumbens and prefrontal cortex neurons produced by previous experience with amphetamine. J Neurosci 17:8491-8497.

Robinson TE, Kolb B (1999) Alterations in the morphology of dendrites and dendritic spines in the nucleus accumbens and prefrontal cortex following repeated treatment with amphetamine or cocaine. Eur J Neurosci 11:1598-1604.

Roitman MF, Schafe GE, Thiele TE, Bernstein IL (1997) Dopamine and sodium appetite: antagonists suppress sham drinking of $\mathrm{NaCl}$ solutions in the rat. Behav Neurosci 111:606-611.

Roitman MF, Patterson TA, Sakai RR, Bernstein IL, Figlewicz DL (1999) Sodium depletion and aldosterone decrease dopamine transporter activity in rat nucleus accumbens but not striatum. Am J Physiol 276:R1339-R1345.

Sakai RR, Fine WB, Epstein AN, Frankmann SP (1987) Salt appetite is enhanced by one prior episode of sodium depletion in the rat. Behav Neurosci 101:724-731

Sakai RR, Frankmann SP, Fine WB, Epstein AN (1989) Prior episodes of sodium depletion increase the need-free sodium intake of the rat. Behav Neurosci 103:186-192.

Segal DS, Mandell AJ (1974) Long-term administration of d-amphetamine: progressive augmentation of motor activity and stereotypy. Pharmacol Biochem Behav 2:249-255.

Wolf G (1982) Refined salt appetite methodology for rats demonstrated by assessing sex differences. J Comp Physiol Psychol 96:10161021. 